\title{
SOLUTION OF TWO VOLTERRA INTEGRAL EQUATIONS
}

\author{
JON C. HELTON
}

Abstract. The letters $f, h, F$ and $G$ denote functions with values in a normed complete ring. With suitable restrictions on these functions, it is established that

$$
f(x)=h(x)+\int_{a}^{x} f(u) G(u, v)\left[\prod^{x}(1+F)\right]
$$

for $a \leqq x \leqq b$ if, and only if,

$$
f(x)=h(x)+\int_{a}^{x} h(u) G(u, v) \cdot\left[\Pi^{x}(1+F+G)\right]
$$

for $a \leqq x \leqq b$, and that

$$
f(x)=h(x)+\int_{a}^{x} f(v) G(u, v)\left[\prod^{x}(1+F)\right]
$$

for $a \leqq x \leqq b$ if, and only if,

$$
f(x)=h(x)+\int_{a}^{x}\left[h(v) \sum_{j=1}^{\infty} G^{j}(u, v)\right]\left[0 \Pi^{x}(1+F)(1-G)^{-1}\right]
$$

for $a \leqq x \leqq b$.

In this paper, product integral techniques for the solution of certain types of Volterra integral equations are developed. These results connect closely with previous integral equation results in papers by $B$. W. Helton [3, 4] and J. C. Helton [7, 8, 9]. In addition, several other papers also contain product integral related techniques for the solution of Volterra integral equations. In particular, the reader is referred to papers by C. W. Bitzer [1, 2], J. V. Herod [11, 12] , D. B. Hinton [13] and J. A. Reneke [16].

In the following, all functions are from $R$ to $N$ or $R \times R$ to $N$, where $R$ denotes the set of real numbers and $N$ denotes a ring which has a multiplicative identity element represented by 1 and a norm $|\cdot|$ with respect to which $N$ is complete and $|1|=1$. Lower case letters are used to denote functions from $R$ to $N$, and upper case letters are used

Received by the editors on February 24, 1976.

AMS(MOS) Subject Classifications. Primary 45N05, Secondary 28A25, 26A39.

Key Words and Phrases. Sum integral, product integral, subdivision-refinement integral, interval function, normed complete ring, Volterra integral equation.

The results contained in this paper are presented in the following abstract: 731-45-15, Solution of two Volterra integral equations. 
to denote functions from $R \times R$ to $N$. Functions on $R \times R$ are assumed to be defined only for elements $\{a, b\}$ of $R \times R$ such that $a<b$.

All integrals and definitions are of the subdivision-refinement type. If $\left\{x_{i}\right\}_{i=0}^{n}$ is a subdivision of $[a, b]$, then $f_{i}=f\left(x_{i}\right)$ and $G_{i}=G\left(x_{i-1}, x_{i}\right)$. The statement that $\int_{a}^{b} G$ exists means there exists an element $L$ of $N$ such that, if $\epsilon>0$, then there exists a subdivision $D$ of $[a, b]$ such that, if $\left\{x_{i}\right\}_{i=0}^{n}$ is a refinement of $D$, then

$$
\left|L-\sum_{i=1}^{n} G_{i}\right|<\epsilon .
$$

Similarly, the statement that $\prod_{a}^{b}(1+G)$ exists means there exists an element $L$ of $N$ such that, if $\epsilon>0$, then there exists a subdivision $D$ of $[a, b]$ such that, if $\left\{x_{i}\right\}_{i=0}^{n}$ is a refinement of $D$, then

$$
\left|L-\prod_{i=1}^{n}\left(1+G_{i}\right)\right|<\epsilon .
$$

Further, the statement that $G$ is in $O M^{*}$ on $[a, b]$ means $(1){ }_{x} \Pi^{y}(1+$ $G)$ exists for $a \leqq x<y \leqq b$ and (2) if $\epsilon>0$, then there exists a subdivision $D$ of $[a, b]$ such that, if $\left\{x_{i}\right\}_{i=0}^{n}$ is a refinement of $D$ and $0 \leqq p<q \leqq n$, then

$$
\left|{ }_{x_{p}} \Pi^{x_{q}}(1+G)-\prod_{i=p+1}^{q}\left(1+G_{i}\right)\right|<\epsilon .
$$

The function $G$ has bounded variation on $[a, b]$ only if there exist a subdivision $D$ of $[a, b]$ and number $B$ such that, if $\left\{x_{i}\right\}_{i=0}^{n}$ is a refinement of $D$, then $\sum_{i=1}^{n}\left|G_{i}\right|<B$. Further, if $\beta>0$, then the notation $|G|<1-\beta$ on $[a, b]$ means there exists a subdivision $D$ of $[a, b]$ such that, if $\left\{x_{i}\right\}_{i=0}^{n}$ is a refinement of $D$ and $1 \leqq i \leqq n$, then $\left|G_{i}\right|<1-\beta$. Also, $G$ is in $O P^{\circ}$ on $[a, b]$ only if there exist a subdivision $D$ of $[a, b]$ and a number $B$ such that, if $\left\{x_{i}\right\}_{i=0}^{n}$ is a refinement of $D$ and $0 \leqq p<$ $q \leqq n$, then

$$
\left|\prod_{i=p+1}^{q}\left(1+G_{i}\right)\right|<B .
$$

The symbols $G\left(p^{-}, p\right), G\left(p^{-}, p^{-}\right), G\left(p, p^{+}\right)$and $G\left(p^{+}, p^{+}\right)$are used to denote $\lim _{x \rightarrow p^{-}}=G(x, p), \lim _{x, y \rightarrow p^{-}} G(x, y), \lim _{x \rightarrow p^{+}} G(p, x)$ and $\lim _{x, y \rightarrow p^{+}} G(x, y)$, respectively. Further, the function $h$ is quasicontinuous on $[a, b]$ only if $\lim _{x \rightarrow p^{-}} h(x)$ exists for $a<p \leqq b$ and $\lim _{x \rightarrow p^{+}} h(x)$ exists for $a \leqq p<b$. For convenience in notation, let $G \in S_{1}$ on $[a, b]$ only if $G\left(p^{-}, p^{-}\right)$exists and is zero for $a<p \leqq b$ and 
$G\left(p^{+}, p^{+}\right)$exists and is zero for $a \leqq p<b$, and let $G \in S_{2}$ on $[a, b]$ only if $G\left(p^{-}, p\right)$ exists for $a<p \leqq b$ and $G\left(p, p^{+}\right)$exists for $a \leqq p<b$.

Additional background on product integration can be obtained in papers by P. R. Masani [15] , J. S. MacNerney [14] , B. W. Helton [3], J. C. Helton [6, 9] and J. C. Helton and S. Stuckwisch [10].

The first integral equation result is now established. Four lemmas are needed.

LEMMA 1. If fand h are functions from $R$ to $N, F$ and $G$ are functions from $R \times R$ to $N,\left\{x_{i}\right\}_{i=0}^{n}$ is a subdivision of $[a, b]$ and

$$
\int_{a}^{x} f(u) G(u, v)\left[v \Pi^{x}(1+F)\right]
$$

exists and is $f(x)-h(x)$ for $a \leqq x \leqq b$, then

$$
\begin{aligned}
f_{m}-h_{m}= & \sum_{i=1}^{m} h_{i-1} G_{i}\left[\prod_{j=i+1}^{m}\left(1+F_{j}+G_{j}\right)\right] \\
& +\sum_{i=1}^{m}\left[c_{i}+d_{i}\right]\left[\prod_{j=i+1}^{m}\left(1+F_{j}+G_{j}\right)\right]
\end{aligned}
$$

for $m=1,2, \cdots, n$, where

$$
c_{i}=\int_{x_{i-1}}^{x_{i}} f(u) G(u, v)\left[{ }^{\prime} \prod^{x_{i}}(1+F)\right]-f_{i-1} G_{i}
$$

and

for $i=1,2, \cdots, n$.

$$
d_{i}=\left[f_{i-1}-h_{i-1}\right]\left[x_{i-1} \Pi^{x_{i}}(1+F)-\left(1+F_{i}\right)\right]
$$

Proof. This result can be established by induction.

LEMMA 2. If $G$ is a function from $R \times R$ to $N$, G has bounded variation on $[a, b]$ and either $\int_{a}^{b} G$ exists or ${ }_{x} \Pi^{y}(1+G)$ exists for $a \leqq x<$ $y \leqq b$, then $G$ is in $O M^{*}$ on $[a, b][6$, Theorem 1, p. 501 and Theorem 4, p. 507].

LEMMA 3. If $S, T$ and $G$ are functions from $R \times R$ to $N$, each of $\mathrm{S}\left(p^{-}, p\right), \mathrm{S}\left(p^{-}, p^{-}\right), T\left(p^{-}, p\right)$ and $T\left(p^{-}, p^{-}\right)$exists for $a<p \leqq b$, each of $\mathrm{S}\left(p, p^{+}\right), \mathrm{S}\left(p^{+}, p^{+}\left(, T\left(p, p^{+}\right)\right.\right.$and $T\left(p^{+}, p^{+}\right)$exists for $a \leqq p<b, G$ has bounded variation on $[a, b]$ and $\int_{x}^{y} G$ exists and is zero for $a \leqq x$ $<y \leqq b$, then $\int_{a}^{b}$ SGT exists and is zero [9, Lemma 1] . 
LEMMA 4. If f and h are functions from $R$ to $N, F$ and $G$ are functions from $R \times R$ to $N, f$ is quasi-continuous on $[a, b], \int_{a}^{b} F$ exists, $\int_{a}^{b} G$ exists, $F$ has bounded variation on $[a, b], G$ has bounded variation on $[a, b]$ and

$$
\int_{a}^{x} f(u) G(u, v)\left[{ }_{v} \prod^{x}(1+F)\right]
$$

exists and is $f(x)-h(x)$ for $a \leqq x \leqq b$, then

$$
\int_{a}^{x} h(u) G(u, v)\left[v \prod^{x}(1+F+G)\right]
$$

exists and is $f(x)-h(x)$ for $a \leqq x \leqq b$.

Proof. Suppose $a \leqq x \leqq b$. If $a=x$, then the desired equality follows immediately. Therefore, suppose $a<x$. We now establish that the desired integral exists and is $f(x)-h(x)$. Let $\epsilon>0$.

If $D$ is a subdivision of $[a, x]$ and $\left\{x_{i}\right\}_{i=0}^{n}$ is a refinement of $D$, then by employing Lemma 1 we have that

$$
\begin{array}{ll} 
& \left|\left\{\sum_{i=1}^{n} h_{i-1} G_{i}\left[x_{i} \prod^{x}(1+F+G)\right]\right\}-\{f(x)-h(x)\}\right| \\
= & \mid\left\{\sum_{i=1}^{n} h_{i-1} G_{i}\left[x_{i} \prod^{x}(1+F+G)\right]\right\} \\
& -\left\{\sum_{i=1}^{n} h_{i-1} G_{i}\left[\prod_{j=i+1}^{n}\left(1+F_{j}+G_{j}\right)\right]\right. \\
& \left.+\sum_{i=1}^{n}\left[c_{i}+d_{i}\right]\left[\prod_{j=i+1}^{n}\left(1+F_{j}+G_{j}\right)\right]\right\} \mid \\
\text { (a) } \leqq & \left|\sum_{i=1}^{n} h_{i-1} G_{i}\left[\prod_{i} \prod^{x}\left(1+F+G_{)}\right)-\prod_{j=i+1}^{n}\left(1+F_{j}+G_{j}\right)\right]\right| \\
\text { (b) } & +\left|\sum_{i=1}^{n} c_{i}\left[\prod_{j=i+1}^{n}\left(1+F_{j}+G_{j}\right)\right]\right| \\
\text { (c) } \quad+\left|\sum_{i=1}^{n} d_{i}\left[\prod_{j=i+1}^{n}\left(1+F_{j}+G_{j}\right)\right]\right|
\end{array}
$$


where $c_{i}$ and $d_{i}$ are defined in the statement of Lemma 1.

It follows from Lemma 2 that $F+G$ is in $O M^{*}$ on $[a, x]$. Hence, there exists a subdivision $D_{1}$ of $[a, x]$ such that, if $\left\{x_{i}\right\}_{i=0}^{n}$ is a refinement of $D_{1}$, then the expression in (a) is less than $\epsilon / 3$.

If $L$ is the function from $R \times R$ to $N$ such that

$$
L(r, s)=\int_{r}^{s} f(u) G(u, v)\left[{ }_{0} \prod^{s}(1+F)-f(r) G(r, s)\right]
$$

for $a \leqq r<s \leqq x$, then $L$ has bounded variation on $[a, x]$ and $\int_{r}^{s} L$ exists and is zero for $a \leqq r<s \leqq x$. Now, since $F+G$ is in $O M^{*}$ on $[a, x]$, it follows from Lemma 3 that there exists a subdivision $D_{2}$ of $[a, x]$ such that, if $\left\{x_{i}\right\}_{i=0}^{n}$ is a refinement of $D_{2}$, then the expression in (b) is less than $\epsilon / 3$.

If $L$ is the function from $R \times R$ to $N$ such that

$$
L(r, s)={ }_{r} \prod s(1+F)-1-F(r, s)
$$

for $a \leqq r<s \leqq x$, then $L$ has bounded variation on $[a, x]$ and $\int_{r}^{s} L$ exists and is zero for $a \leqq r<s \leqq x$. Now, since $f$ and $h$ are quasicontinuous on $[a, x]$ and $F+G$ is in $O M^{*}$ on $[a, x]$, it follows from Lemma 3 that there exists a subdivision $D_{3}$ of $[a, x]$ such that, if $\left\{x_{i}\right\}_{i=0}^{n}$ is a refinement of $D_{3}$, then the expression in (c) is less than $\epsilon / 3$.

Let $D$ denote the subdivision $D_{1} \cup D_{2} \cup D_{3}$ of $[a, x]$. Thus, if $\left\{x_{i}\right\}_{i=0}^{n}$ is a refinement of $D$, then it follows from the definitions of $D_{1}$, $D_{2}$ and $D_{3}$ that

$$
\begin{gathered}
\left|\left\{\sum_{i=1}^{n} h_{i-1} G_{i}\left[x_{i} \prod^{x}(1+F+G)\right]\right\}-\{f(x)-h(x)\}\right| \\
<\epsilon / 3+\epsilon / 3+\epsilon / 3=\epsilon .
\end{gathered}
$$

Therefore, the desired integral exists and is $f(x)-h(x)$.

THeOREM 1. If $f$ and $h$ are functions from $R$ to $N, F$ and $G$ are functions from $R \times R$ to $N, \int_{a}^{b} F$ exists, $\int_{a}^{b} G$ exists, $F$ has bounded variation on $[a, b]$ and $G$ has bounded variation on $[a, b]$, then the following statements are equivalent:

(1) fis quasi-continuous on $[a, b]$ and

$$
\int_{a}^{x} f(u) G(u, v)\left[v \Pi^{x}(1+F)\right]
$$

exists and is $f(x)-h(x)$ for $a \leqq x \leqq b$, and 
(2) $h$ is quasi-continuous on $[a, b]$ and

$$
\int_{a}^{x} h(u) G(u, v)\left[v \prod^{x}(1+F+G)\right]
$$

exists and is $f(x)-h(x)$ for $a \leqq x \leqq b$.

Proof. [(1) $\rightarrow(2)]$. Suppose (1) is true. Then it follows immediately that $h$ is quasi-continuous on $[a, b]$. Also, the hypothesis of Lemma 4 is satisfied. Hence, the desired integral exists and is $f(x)-$ $h(x)$ for $a \leqq x \leqq b$. Therefore, (1) implies (2).

$[(2) \rightarrow(1)]$. Suppose (2) is true. Then, it follows immediately that $f$ is quasi-continuous on $[a, b]$. Further, it also follows that $\int_{a}^{x} h(u)$ $[-G(u, v)]\left[{ }_{v} \Pi^{x}(1+f+G)\right]$ exists and is $h(x)-f(x)$ for $a \leqq x \leqq b$. Now, by applying Lemma 4 , we have that $\int_{a}^{x} f(u)[-G(u, v)]\left[\Pi^{x}(1+\right.$ $F+G-G)]$ exists and is $h(x)-f(x)$ for $a \leqq x \leqq b$. Hence, the desired integral exists and is $f(x)-h(x)$ for $a \leqq x \leqq b$. Therefore, (2) implies (1).

The proot of Theorem 1 is now complete. The second integral equation result is established next. Two additional lemmas are needed.

LEMMA 5. If $\beta>0, f$ and $h$ are functions from $R$ to $N, F$ and $G$ are functions from $R \times R$ to $N,\left\{x_{i}\right\}_{i=0}^{n}$ is a subdivision of $[a, b]$ such that $\left|G_{i}\right|<1-\beta$ for $i=1,2, \cdots, n$ and

$$
\int_{a}^{x} f(v) G(u, v)\left[v \Pi^{x}(1+F)\right]
$$

exists and is $f(x)-h(x)$ for $a \leqq x \leqq b$, then

$$
\begin{aligned}
f_{m}-h_{m}= & \sum_{i=1}^{m}\left[h_{i} \sum_{j=1}^{\infty} G_{i}{ }^{j}\right]\left[\prod_{k=i+1}^{m}\left(1+F_{k}\right)\left(1-G_{k}\right)^{-1}\right] \\
& +\sum_{i=1}^{m}\left[\left(c_{i}+d_{i}\right)\left(1-G_{i}\right)^{-1}\right]\left[\prod_{k=i+1}^{m}\left(1+F_{k}\right)\left(1-G_{k}\right)^{-1}\right]
\end{aligned}
$$

for $m=1,2, \cdots, n$, where

$$
c_{i}=\int_{x_{i-1}}^{x_{i}} f(v) G(u, v)\left[0 \prod^{x_{i}}(1+F)\right]-f_{i} G_{i}
$$

and

for $i=1,2, \cdots, n$.

$$
d_{i}=\left[f_{i-1}-h_{i-1}\right]\left[{ }_{x_{i-1}} \prod^{x_{i}}(1+F)-\left(1+F_{i}\right)\right]
$$


Proof. This result can be established by induction.

LeMMA 6. If $\beta>0, f$ and $h$ are functions from $R$ to $N, F$ and $G$ are functions from $R \times R$ to $N, f$ is quasi-continuous on $[a, b], \int_{a}^{b} F$ exists, $\int_{a}^{b} G$ exists, $F$ has bounded variation on $[a, b], G$ has bounded variation on $[a, b],|G|<1-\beta$ on $[a, b]$ and

$$
\int_{a}^{x} f(v) G(u, v)\left[\prod^{x}(1+F)\right]
$$

exists and is $f(x)-h(x)$ for $a \leqq x \leqq b$, then

$$
\int_{a}^{x}\left[h(v) \sum_{j=1}^{\infty} G^{j}(u, v)\right]\left[v \prod^{x}(1+F)(1-G)^{-1}\right]
$$

exists and is $f(x)-h(x)$ for $a \leqq x \leqq b$.

Proof. Through the use of Lemma 5 instead of Lemma 1, this result can be established by a proof similar to the proof used to establish Lemma 4.

THEOREM 2. If $\beta>0, f$ and $h$ are functions from $R$ to $N, F$ and $G$ are functions from $R \times R$ to $N, \int_{a}^{b} F$ exists, $\int_{a}^{b} G$ exists, $F$ has bounded variation on $[a, b], G$ has bounded variation on $[a, b]$ and $|G|<$ $1-\beta$ on $[a, b]$, then the following statements are equivalent:

(1) fis quasi-continuous on $[a, b]$ and

$$
\int_{a}^{x} f(v) G(u, v)\left[v \Pi^{x}(1+F)\right]
$$

exists and is $f(x)-h(x)$ for $a \leqq x \leqq b$, and

(2) $h$ is quasi-continuous on $[a, b]$ and

$$
\int_{a}^{x}\left[h(v) \sum_{j=1}^{\infty} G^{j}(u, v)\right]\left[0 \Pi^{x}(1+F)(1-G)^{-1}\right]
$$

exists and is $f(x)-h(x)$ for $a \leqq x \leqq b$.

Proof. Through the use of Lemma 6 instead of Lemma 4, this result can be established by a proof similar to the proof used to establish Theorem 1.

REMARK. The conditions on the function $F$ in Theorems 1 and 2 are actually more stringent than what is needed to establish the equivalence of the two statements in their conclusions. These theorems remain true if the requirements that " $\int_{a}^{b} F$ exists and $F$ has bounded variation on $[a, b]$ " are replaced by the weaker requirements that " $F$ is in $O M^{*}$, 
$S_{1} \cap S_{2}$ and $O P^{\circ}$ on $[a, b], \int_{r}^{s} L$ exists and is zero for $a \leqq r<s \leqq b$ and $L$ has bounded variation on $[a, b]$, where $L$ denotes the interval function such that

$$
L(r, s)={ }_{r} \prod^{s}(1+F)-1-F(r, s)
$$

for $a \leqq r<s \leqq b$." However, I decided not to complicate the statement and proof of these results by using the more general conditions on F.

When $F$ is supposed to satisfy these more general conditions, the major complication is the need to establish that $F+G$ is in $O M^{*}$ and $O P^{\circ}$ on $[a, b]$. That $F+G$ does indeed satisfy these conditions can be established by using results from a previous paper by J. C. Helton [5, Theorem 1, p. 355 and Lemma 5, p. 358] .

\section{BiBLIOGRAPHY}

1. C. W. Bitzer, Stieltjes-Volterra integral equations, Illinois J. Math. 14 (1970), 434-451.

2. - Convolution, fixed point, and approximation in Stieltjes-Volterra integral equations, J. Australian Math. Soc. 14 (1972), 182-199.

3. B. W. Helton, Integral equations and product integrals, Pacific J. Math. 16 (1966), 297-322.

4. —_ Solutions of $f(x)=f(a)+(R L) \int_{a}^{x}(f H+f G)$ for rings, Proc. Amer. Math. Soc. 25 (1970), 735-742.

5. J. C. Helton, Mutual existence of product integrals in normed rings, Trans. Amer. Math. Soc. 211 (1975), 353-363.

6. - Mutual existence of sum and product integrals, Pacific J. Math. 56 (1975), 495-516.

7. — Product integrals and the solution of integral equations, Pacific J. Math. 58 (1975), 87-103.

8. - Solution of integral equations by product integration, Proc. Amer. Math. Soc. 49 (1975), 401-406.

9. - Existence of integrals and the solution of integral equations, Trans. Amer. Math. Soc. 229 (1977), 307 327.

10. J. C. Helton and S. Stuckwisch, Numerical approximation of product integrals, J. Math. Anal. Appl. 56 (1976), 410-437.

11. J. V. Herod, Multiplicative inverses of solutions for Volterra-Stieltjes integral equations, Proc. Amer. Math. Soc. 22 (1969), 650-656.

12. - A pairing of a class of evolution systems with a class of generators, Trans. Amer. Math. Soc. 157 (1971), 247-260.

13. D. B. Hinton, A Stieltjes-Volterra integral equation theory, Can. J. Math. 18 (1966), 314-331.

14. J. S. MacNerney, Integral equations and semigroups, Illinois J. Math. 7 (1963), 148-173.

15. P. R. Masani, Multiplicative Riemann integration in normed rings, Trans. Amer. Math. Soc. 61 (1947), 147-192.

16. J. A. Reneke, A product integral solution of a Stieltjes-Volterra integral equation, Proc. Amer. Math. Soc. 24 (1970), 621-626.

Arizona State University, Tempe, Arizona 85281 